\title{
Enabling Depositor-initiated PDB coordinate replacement through file versioning
}

Christine Zardecki ${ }^{a}$, Jasmine Young ${ }^{a}$, John Westbrook ${ }^{a}$, Zukang Fenga ${ }^{a}$ Ezra Peisach ${ }^{a}$, Irina Persikovaa, Yuhe Liang ${ }^{\mathrm{a}}$, Aleks Gutmanas ${ }^{\mathrm{b}}$, John Berrisford ${ }^{\mathrm{b}}$, Yasuyo Ikegawac, Minyu Chen ${ }^{\mathrm{c}}$, Stephen K. Burley ${ }^{\mathrm{a}, \mathrm{d}, \mathrm{e}}$, Sameer Valenkar ${ }^{\mathrm{b}}$, Genji Kurisu ${ }^{\mathrm{c}}$

${ }^{a}$ RCSB PDB, Institute for Quantitative Biomedicine, Rutgers, The State University of New Jersey, Piscataway, NJ 08854, United States, Christine.zardecki@rcsb.org ${ }^{b} P D B e$, EMBL-European Bioinformatics Institute, Hinxton, United Kingdom 'PDBj, Institute for Protein Research, Osaka University, Osaka, Japan ${ }^{\mathrm{d}}$ RCSB PDB, Skaggs School of Pharmacy and Pharmaceutical Sciences and San Diego Supercomputer Center, University of California San Diego, La Jolla, CA 92093, United States. ${ }^{e}$ Rutgers Cancer Institute of New Jersey, New Brunswick, NJ 08903

wwPDB has introduced a versioning system to enable depositor-initiated or wwPDB-initiated updates to previously released PDB entries while retaining the same PDB accession code.

Changes made to entries during versioning are considered to be either "major" or "minor". Updates to atomic coordinates, polymer sequence, or chemical description trigger a major version increment. Other changes are indicated as "minor".

The most recent version of the entry will be available in the main PDB archive FTP (ftp.wwpdb.org) and the versioned FTP archive (ftp-versioned.wwpdb.org). All major versions of a PDB structure will be retained in the versioned FTP archive. Any changes introduced during versioning are recorded in PDBx/mmCIF audit categories for entry revision.

For versioned entries, changes will not be made to the PDB ID or the deposited experimental data.

Depositors can begin to submit new coordinates for entries originally submitted via the OneDep system by Fall 2019. Requests should be initiated using the OneDep communication panel for the entry in question. Entries will be processed and released immediately after author approval.

In the future, depositors will be able to submit coordinates for all entries, including those deposited using legacy systems (e.g., AutoDep, ADIT).

wwPDB members are RCSB PDB (supported by NSF, NIH, and DOE), PDBe (EMBL-EBI, Wellcome Trust, BBSRC, MRC, and EU), and PDBj (NBDC-JST), and BMRB (NIGMS). 